\title{
COUNTEREXAMPLES TO BILINEAR ESTIMATES RELATED WITH THE KDV EQUATION AND THE NONLINEAR SCHRÖDINGER EQUATION*
}

\author{
Kenji Nakanishi ${ }^{\dagger}$ Hideo TakaOKa $^{\ddagger}$ and Yoshio Tsutsumi ${ }^{\S}$
}

1. Introduction and main results. The solvability of nonlinear evolution equations with quadratic nonlinearity is often reduced to the bilinear estimates corresponding to their nonlinearity. In other words, we can prove a new existence theorem of solution for the Cauchy problem of the quadratic nonlinear evolution equation, if we have a new bilinear estimate to control the nonlinearity. For nonlinear dispersive wave equations such as the nonlinear Schrödinger equation and the KdV equation, there has been a great progress in this direction. In [23], Strichartz did not only prove new space-time integrability estimates for the Schrödinger and the wave equations but also he pointed out that they were equivalent to the Fourier restriction theorem. Nowadays these space-time estimates are called the Strichartz estimates and it is well known that the Strichartz estimates are useful for the study of nonlinear dispersive wave equations. In [3] and [4], Bourgain further developed the Strichartz estimate and introduced the Fourier restriction norm method to solve the Cauchy problem of the nonlinear Schrödinger equation and the KdV equation in much weaker spaces than before. He directly evaluated the nonlinear term by using the argument in the proof of the Fourier restriction theorem (for the Fourier restriction theorem, see, e.g., Tomas [27]). One of the new ingredients in his papers [3] and [4] is a set of new bilinear estimates corresponding to the nonlinearity (see, e.g., Lemmas 7.41 and 7.42 in [4]). These bilinear estimates effectively represent a kind of smoothing property produced by the oscillation of wave. For the quadratic nonlinearity, Bourgain's argument is considered as a more powerful machine than the Strichartz estimate. In [9], [10] and [11], Kenig, Ponce and Vega simplified Bourgain's proof and improved the bilinear estimates. Recently, this method has been applied to various nonlinear evolution equations (see, e.g., [1], [2], [5], [7], [8], [15], [22], [24]-[26] and [28]-[30]). The related method for nonlinear wave equations was developed by Klainerman and Machedon [12]-[14] (see also Rauch and Reed [18]). In the present paper, we consider the optimality of the bilinear estimates obtained in [10] and [11].

We now list the notations, which will be used throughout this paper. For $f(t, x) \in$ $\mathcal{S}^{\prime}\left(\mathbf{R}^{2}\right)$, we denote the Fourier transform in $t$ and $x$ of $f$ by $\hat{f}$. For $b, s \in \mathbf{R}$, we define the spaces $X_{b, s}$ and $Y_{b, s}$ as follows.

$$
\begin{aligned}
& X_{b, s}=\left\{f \in \mathcal{S}^{\prime}\left(\mathbf{R}^{2}\right) ; \quad\|f\|_{X_{b, s}}<\infty\right\}, \\
& Y_{b, s}=\left\{f \in \mathcal{S}^{\prime}\left(\mathbf{R}^{2}\right) ; \quad\|f\|_{Y_{b, s}}<\infty\right\},
\end{aligned}
$$

\footnotetext{
* Dedicated to Professor Seiji Ukai on the occasion of his 60th birthday.

† Graduate School of Mathematics, Nagoya University, Nagoya 464-8602, Japan.

$\ddagger$ Department of Mathematics, Hokkaido University, Sapporo 060-0810, Japan.

$\S$ Mathematical Institute, Tohoku University, Sendai 980-8578, Japan.
} 
where

$$
\begin{aligned}
\|f\|_{X_{b, s}} & =\left(\int_{\mathbf{R}^{2}}\left(1+\left|\tau-\xi^{3}\right|\right)^{2 b}(1+|\xi|)^{2 s}|\hat{f}(\tau, \xi)|^{2} d \tau d \xi\right)^{1 / 2}, \\
\|f\|_{Y_{b, s}} & =\left(\int_{\mathbf{R}^{2}}\left(1+\left|\tau-\xi^{2}\right|\right)^{2 b}(1+|\xi|)^{2 s}|\hat{f}(\tau, \xi)|^{2} d \tau d \xi\right)^{1 / 2} .
\end{aligned}
$$

For $z \in \mathbf{C}$, we denote the complex conjugate of $z$ by $\bar{z}$. We put

$$
\langle f, g\rangle=\int_{\mathbf{R}^{2}} f(t, x) \overline{g(t, x)} d t d x .
$$

For two functions $f(t, x)$ and $g(t, x)$, let $f * g$ denote the convolution with respect to the time and space variables. Let $D=\mathcal{F}^{-1}|\xi| \mathcal{F}$, where $\mathcal{F}$ and $\mathcal{F}^{-1}$ denote the Fourier transform and the inverse Fourier transform, respectively. For two functions $P\left(a_{1}, \cdots, a_{n}\right)$ and $Q\left(a_{1}, \cdots, a_{n}\right)$ with variables $\left(a_{1}, \cdots, a_{n}\right) \in \mathbf{R}^{n}$, we write $P \sim Q$ if there exists a positive constant $C$ such that

$$
C^{-1}\left|Q\left(a_{1}, \cdots, a_{n}\right)\right| \leq\left|P\left(a_{1}, \cdots, a_{n}\right)\right| \leq C\left|Q\left(a_{1}, \cdots, a_{n}\right)\right|
$$

as $\left|a_{1}\right|+\cdots+\left|a_{n}\right| \rightarrow \infty$.

In [10] and [11], Kenig, Ponce and Vega proved the following theorem (see Theorems 1.1 and 1.3 in [10] and Theorems 1.1-1.4 in [11]).

Theorem 0. (i) For any $s \in(-3 / 4,0]$, there exist $b \in(1 / 2,1)$ and $C>0$ such that

$$
\|D(u v)\|_{X_{b-1, s}} \leq C\|u\|_{X_{b, s}}\|v\|_{X_{b, s}} .
$$

Furthermore, for any $s<-3 / 4$ and any $b \in \mathbf{R}$, the estimate (1.1) fails.

(ii) For any $s \in(-3 / 4,0]$, there exist $b \in(1 / 2,1)$ and $C>0$ such that

$$
\begin{aligned}
\|u v\|_{Y_{b-1, s}} & \leq C\|u\|_{Y_{b, s}}\|v\|_{Y_{b, s}}, \\
\|\bar{u} \bar{v}\|_{Y_{b-1, s}} & \leq C\|u\|_{Y_{b, s}}\|v\|_{Y_{b, s}} .
\end{aligned}
$$

For any $s \in(-1 / 4,0]$, there exist $b \in(1 / 2,1)$ and $C>0$ such that

$$
\|u \bar{v}\|_{Y_{b-1, s}} \leq C\|u\|_{Y_{b, s}}\|v\|_{Y_{b, s}} .
$$

Furthermore, for any $s<-3 / 4$ and any $b \in \mathbf{R}$, the estimates (1.2) and (1.3) fail, and for any $s<-1 / 4$ and any $b \in \mathbf{R}$, the estimate (1.4) fails.

The estimate (1.1) leads to the local well-posedness of the Cauchy problem for the $\mathrm{KdV}$ equation in $H^{s}$ with $s>-3 / 4$ :

$$
\begin{aligned}
& \frac{\partial u}{\partial t}+\frac{\partial^{3} u}{\partial x^{3}}+u \frac{\partial u}{\partial x}=0, \quad t \in[-T, T], \quad x \in \mathbf{R}, \\
& u(0, x)=u_{0}(x), \quad x \in \mathbf{R} .
\end{aligned}
$$

The estimates (1.2), (1.3) and (1.4) also lead to the local well-posedness of the Cauchy problem for the following quadratic nonlinear Schrödinger equations in $H^{s}$ with $s>$ $-3 / 4, s>-3 / 4$ and $s>-1 / 4$, respectively:

$$
\begin{aligned}
& i \frac{\partial u}{\partial t}+\frac{\partial^{2} u}{\partial x^{2}}=F_{j}(u, u), \quad t \in[-T, T], \quad x \in \mathbf{R}, \quad j=1,2,3 \\
& u(0, x)=u_{0}(x), \quad x \in \mathbf{R}
\end{aligned}
$$


where

$$
F_{1}(u, u)=u^{2}, \quad F_{2}(u, u)=\bar{u}^{2}, \quad F_{3}(u, u)=u \bar{u} .
$$

However, it has been open whether the estimates (1.1)-(1.4) hold for critical indices $s=-3 / 4, s=-3 / 4, s=-3 / 4$ and $s=-1 / 4$, respectively. This problem is interesting and important from both viewpoints of partial differential equations and harmonic analysis. In this paper, we show that the estimates (1.1)-(1.4) break down for these critical indices $s$. We have the following theorem.

THEOREM 1.

(i) Let $s=-3 / 4$. For any $b \in \mathbf{R}$, the estimate (1.1) fails.

(ii) Let $s=-3 / 4$. For any $b \in \mathbf{R}$, the estimates (1.2) and (1.3) fail.

(iii) Let $s=-1 / 4$. For any $b$ with $b \geq 1 / 2$, the estimate (1.4) fails.

REMARK 1.

(i) Theorem 1 (i) shows that the $X_{b, s}$ space is not sufficient for the proof of the local well-posedness in $H^{s}, s=-3 / 4$ of the $\mathrm{KdV}$ equation. But it does not necessarily imply the ill-posedness in $H^{s}, s=-3 / 4$ of the $\mathrm{KdV}$ equation. This is also the case with Theorem 1 (ii), when we consider (1.7)-(1.8) with $j=1,2$ in $H^{s}, s=-3 / 4$.

(ii) In fact, a stronger result holds than Theorem 1 (iii). That is, if $a \geq-1 / 2$, for any $\alpha, \beta \in \mathbf{R}$, the following estimate fails:

$$
\|u \bar{v}\|_{Y_{a,-1 / 4}} \leq C\|u\|_{Y_{\alpha,-1 / 4}}\|v\|_{Y_{\beta,-1 / 4}} .
$$

There may be a chance that the estimate (1.4) holds with $s=-1 / 4$ and $b<1 / 2$. But, even if this were true, it would be difficult that we apply this estimate to (1.7)-(1.8) with $j=3$.

We conclude this section by giving a few remarks on the results for nonlinear wave equations, which are closely related to our problem. For nonlinear wave equations, the time local well-posedness in minimal regularity has been extensively studied (see, e.g., [8], [12]-[21], [26], [29] and [30]). In this context, not only have the bilinear estimates corresponding to nonlinearity been studied, but also sharp counterexamples are constructed in [16] and [17], which show actually the ill-posedness of the Cauchy problem in critical regularity.

The plan of this paper is the following. In Section 2, we give a proof of Theorem 1 (i). In Section 3, we show Theorem 1 (ii) and (iii).

2. Proof of Theorem 1 (i). In this section we give a proof of Theorem 1 (i). The proof of Theorem 1.3 in [10] implies that if the estimate (1.1) holds with $s=-3 / 4$, we must have $b=1 / 2$ (see the relations (4.8) and (4.15) in [10]). Therefore, we have only to consider the case of $s=-3 / 4$ and $b=1 / 2$.

Let $N$ and $m$ be sufficiently large positive integers such that $4^{m+1} \ll N$. We put 
$A_{j}, j=0,1, \cdots, m$ as follows.

$$
\begin{aligned}
A_{m}= & \left\{(\tau, \xi) \in \mathrm{R}^{2} ;(\tau, \xi)\right. \text { belongs to the inside of two parallelograms with } \\
& \text { vertices }\left(N^{3}-4^{m}, N\right),\left(N^{3}-4^{m+1}, N\right), \\
& \left(N^{3}-4^{m+1}+3 N^{3 / 2} 4^{(m+1) / 2}, N+4^{(m+1) / 2} N^{-1 / 2}\right), \\
& \left(N^{3}-4^{m}+3 N^{3 / 2} 4^{(m+1) / 2}, N+4^{(m+1) / 2} N^{-1 / 2}\right) \\
\text { and }\left(-N^{3}+4^{m},-N\right),\left(-N^{3}+4^{m+1},-N\right), & \\
& \left(-N^{3}+4^{m+1}-3 N^{3 / 2} 4^{(m+1) / 2},-N-4^{(m+1) / 2} N^{-1 / 2}\right), \\
& \left.\left(-N^{3}+4^{m}-3 N^{3 / 2} 4^{(m+1) / 2},-N-4^{(m+1) / 2} N^{-1 / 2}\right)\right\}, \\
A_{m-1}= & \left\{(\tau, \xi) ; N \leq|\xi| \leq N+4^{(m+1) / 2} N^{-1 / 2}, 4^{m-1} \leq\left|\tau-\xi^{3}\right|<4^{m},\right. \\
A_{j}= & \left\{(\tau, \xi) ; N \leq|\xi| \leq N+4^{(m+1) / 2} N^{-1 / 2}, 4^{j} \leq\left|\tau-\xi^{3}\right|<4^{j+1}\right\}, \\
&
\end{aligned}
$$

Let $R$ denote the region consisting of two parallelograms similar to the parallelograms in the definition of $A_{m}$ with one fourth area, which are centered at the points $\left(0,-\frac{7}{12} 4^{(m+1) / 2} N^{-1 / 2}\right)$ and $\left(0, \frac{7}{12} 4^{(m+1) / 2} N^{-1 / 2}\right)$, respectively, and whose longest sides are parallel to the vector $\left(3 N^{3 / 2} 4^{(m+1) / 2}, 4^{(m+1) / 2} N^{-1 / 2}\right)$. Here we note that the set $A_{m}$ translated by a vector of $R$ intersects effectively each $A_{j}, 0 \leq j \leq m$. For a measurable set $A \subset \mathbf{R}^{2}$, we denote the area of $A$ and the characteristic function of $A$ by $|A|$ and $\chi_{A}$, respectively. By the definitions of the sets $A_{j}$ and $R$, we have

$$
\begin{aligned}
&\left|A_{j}\right|=2 \int_{N}^{N+4^{m / 2} N^{-1 / 2}} \quad\left[\left(\xi^{3}-4^{j}\right)-\left(\xi^{3}-4^{j+1}\right)\right] d \xi \\
& \sim 4^{j} 4^{m / 2} N^{-1 / 2}, \quad 0 \leq j \leq m-1, \\
&\left|A_{m}\right| \sim 4^{m} \sqrt{\frac{4^{m+1}}{N}} \sim 4^{3 m / 2} N^{-1 / 2}, \\
&|R| \sim 4^{3 m / 2} N^{-1 / 2}, \\
& \chi_{A_{j}} * \chi_{A_{m}} \geq C\left|A_{j}\right| \chi_{R}, \quad 0 \leq j \leq m, \\
& j \neq k
\end{aligned}
$$

Let $\left\{a_{j}\right\}_{j=0}^{m}$ be an arbitrary sequence such that $a_{j} \geq 0$ for $0 \leq j \leq m$. We put

$$
\hat{u}=N \sum_{0 \leq j \leq m} 4^{-j-m / 4} a_{j} \chi_{A_{j}} .
$$


Then, a simple calculation yields

$$
\begin{aligned}
\hat{u} * \hat{u} & =N^{2}\left(\sum_{0 \leq j \leq m} 4^{-j-m / 4} a_{j} \chi_{A_{j}}\right) *\left(\sum_{0 \leq k \leq m} 4^{-k-m / 4} a_{k} \chi_{A_{k}}\right) \\
= & N^{2} \sum_{0 \leq k \leq m} \sum_{0 \leq j \leq k} 4^{-j-k-m / 2} a_{j} a_{k} \chi_{A_{j}} * \chi_{A_{k}} \\
& +N^{2} \sum_{0 \leq k \leq m} \sum_{k<j \leq m} 4^{-j-k-m / 2} a_{j} a_{k} \chi_{A_{j}} * \chi_{A_{k}} \\
\geq & N^{2} \sum_{k=m} \sum_{0 \leq j \leq k} 4^{-j-k-m / 2} a_{j} a_{k} \chi_{A_{j}} * \chi_{A_{k}} \\
= & N^{2} a_{m}\left(\sum_{0 \leq j \leq m} 4^{-j-3 m / 2} a_{j} \chi_{A_{j}}\right) * \chi_{A_{m}} .
\end{aligned}
$$

By (2.1)-(2.6), we obtain

$$
\begin{aligned}
\| & || \xi \mid(1+|\xi|)^{-3 / 4}\left(1+\left|\tau-\xi^{3}\right|\right)^{-1 / 2} \hat{u} * \hat{u} \|_{L^{2}\left(\mathbf{R}^{2}\right)} \\
\geq & C\left[\left(4^{m+1} N^{-1}\right)\left(4^{-(m+1) / 2} N^{-3 / 2}\right) N^{4} a_{m}^{2}\right. \\
& \left.\times \int_{\mathbf{R}^{2}}\left(\sum_{0 \leq j \leq m} 4^{-j-3 m / 2} a_{j}\left(\chi_{A_{j}} * \chi_{A_{m}}\right)\right)^{2} d \tau d \xi\right]^{1 / 2} \\
\geq C[ & \left.4^{-5 m / 2} N^{3 / 2} a_{m}^{2}\left(\sum_{0 \leq j \leq m} 4^{-j} 4^{j} 4^{m / 2} N^{-1 / 2} a_{j}\right)^{2} 4^{3 m / 2} N^{-1 / 2}\right]^{1 / 2} \\
\geq & C a_{m}\left(\sum_{0 \leq j \leq m} a_{j}\right), \\
\| & (1+|\xi|)^{-3 / 4}\left(1+\left|\tau-\xi^{3}\right|\right)^{1 / 2} \hat{u} \|_{L^{2}\left(\mathbf{R}^{2}\right)} \\
= & \left(\int_{\mathbf{R}^{2}}(1+|\xi|)^{-3 / 2}\left(1+\left|\tau-\xi^{3}\right|\right) N^{2} \sum_{0 \leq j \leq m} 4^{-2 j-m / 2} a_{j}^{2} \chi_{A_{j}} d \tau d \xi\right)^{1 / 2} \\
\leq & C\left(N^{2} \sum_{0 \leq j \leq m} 4^{-2 j-m / 2} N^{-3 / 2} 4^{j} a_{j}^{2}\left|A_{j}\right|\right)^{1 / 2} \\
\leq & C\left(N^{1 / 2} \sum_{0 \leq j \leq m} 4^{-j-m / 2} 4^{j} 4^{m / 2} N^{-1 / 2} a_{j}^{2}\right)^{1 / 2} \\
\leq & C\left(\sum_{0 \leq j \leq m} a_{j}^{2}\right)^{1 / 2} .
\end{aligned}
$$

Therefore, if the estimate (1.1) holds with $s=-3 / 4$ and $b=1 / 2$, then we conclude by (2.7) and (2.8) that the following inequality must hold.

$$
a_{m}\left(\sum_{0 \leq j \leq m} a_{j}\right) \leq C\left(\sum_{0 \leq j \leq m} a_{j}^{2}\right)
$$

where $C$ is a positive constant independent of $m$ and $N$. We now choose sequences 
$\left\{a_{j}^{(m)}\right\}_{j=0}^{m}, m=1,2, \cdots$ such that $a_{j}^{(m)} \geq 0, a_{m}^{(m)}=1$ and

$$
\begin{aligned}
& \sup _{m \geq 1} \sum_{0 \leq j \leq m}\left(a_{j}^{(m)}\right)^{2}<\infty, \\
& \sum_{0 \leq j \leq m} a_{j}^{(m)} \longrightarrow \infty \quad(m \rightarrow \infty) .
\end{aligned}
$$

For example, we can choose $a_{j}^{(m)}=(1+j)^{-1}, j=0,1, \cdots, m-1$ and $a_{m}^{(m)}=1$. We insert $\left\{a_{j}^{(m)}\right\}$ into the inequality (2.9) and let $m \rightarrow \infty$ in the resulting inequality to obtain a contradiction. This completes the proof of Theorem 1 (i).

REMARK 2. The above proof shows that when $s=-3 / 4$ and $b=1 / 2$, the breakdown of the estimate (1.1) is caused by the function $\hat{u}$ with its support in the region distant from the curve $\left\{\tau=\xi^{3}\right\}$ in contrast to the other case (see the proof of Theorem 1.3 in [10]).

3. Proof of Theorem 1 (ii) and (iii). In this section, we show Theorem 1 (ii) and (iii).

Proof of Theorem 1 (ii). We only prove that when $s=-3 / 4$, the estimate (1.2) fails for any $b \in \mathbf{R}$. Because the failure of (1.3) with $s=-3 / 4$ can be proved in the same way. By the duality argument and the Plancherel theorem, the estimate (1.2) is equivalent to the following:

$$
\langle\hat{w}, \hat{u} * \hat{v}\rangle \leq C\|w\|_{Y_{1-b,-s}}\|u\|_{Y_{b, s}}\|v\|_{Y_{b, s}} .
$$

The proof of Theorem 1.4 (i) in [11] implies that if the estimate (3.1) holds with $s=-3 / 4$, we must have $b=1 / 2$ (see the relations (2.59) and (2.62) in [11]). Therefore, we have only to consider the case of $s=-3 / 4$ and $b=1 / 2$. We can construct a counterexample similar to that in Section 2, but we here present a counterexample of slightly different type.

Let $N$ be a sufficiently large positive integer and let $\eta$ be a sufficiently small positive number independent of $N$. We define three functions $\hat{u}, \hat{v}$ and $\hat{w}$ as follows.

$$
\begin{aligned}
& \hat{u}(\tau, \xi)= \begin{cases}1, & -N \leq \xi \leq-N-1, \quad\left|\tau-\xi^{2}\right| \leq 1, \\
0, & \text { otherwise },\end{cases} \\
& \hat{v}(\tau, \xi)= \begin{cases}1, & N \leq \xi \leq N+1, \quad\left|\tau+\xi^{2}\right| \leq 1, \\
0, & \text { otherwise }\end{cases} \\
& \hat{w}(\tau, \xi)= \begin{cases}(1+\tau)^{-1}, \quad|\tau+2 N \xi| \leq \eta, & 1 \leq \tau \leq \eta N, \\
0, & \text { otherwise }\end{cases}
\end{aligned}
$$

By the definitions of $\hat{u}$ and $\hat{v}$, we have

$$
\operatorname{supp}(\hat{u} * \hat{v}) \supset\{(\tau, \xi) ;|\tau+2 N \xi| \leq \eta, \quad 1 \leq \tau \leq \eta N\}
$$

Therefore,

$$
\begin{gathered}
\langle\hat{w}, \hat{u} * \hat{v}\rangle \geq C \int_{1}^{\eta N}(1+\tau)^{-1} \int_{-\tau /(2 N)-\eta /(2 N)}^{-\tau /(2 N)+\eta /(2 N)} d \xi d \tau \\
\sim N^{-1} \int_{1}^{\eta N}(1+\tau)^{-1} d \tau \sim N^{-1} \log N
\end{gathered}
$$


On the other hand, since $|\xi| \leq C$ for $(\tau, \xi) \in$ supp $\hat{w}$, simple calculations yield

$$
\begin{aligned}
&\|w\|_{Y_{1 / 2,3 / 4}} \sim\left(\int_{1}^{\eta N} \int_{-\tau /(2 N)-\eta /(2 N)}^{-\tau /(2 N)+\eta /(2 N)}(1+\tau)^{-2}(1+|\xi|)^{3 / 2}\right. \\
&\left.\quad \times\left(1+\left|\tau-\xi^{2}\right|\right) d \xi d \tau\right)^{1 / 2} \\
& \sim N^{-1 / 2}\left(\int_{1}^{\eta N}(1+\tau)^{-1} d \tau\right)^{1 / 2} \\
& \sim N^{-1 / 2}(\log N)^{1 / 2} \\
&\|u\|_{Y_{1 / 2,-3 / 4}} \sim N^{-3 / 4} \\
&\|v\|_{Y_{1 / 2,-3 / 4}} \sim N^{-3 / 4} N \sim N^{1 / 4} .
\end{aligned}
$$

If the estimate (3.1) holds with $s=-3 / 4$ and $b=1 / 2$, we must obtain by (3.2)-(3.5)

$$
\begin{aligned}
N^{-1} \log N & \leq C N^{-1 / 2}(\log N)^{1 / 2} \times N^{-3 / 4} \times N^{1 / 4} \\
& =C N^{-1}(\log N)^{1 / 2}
\end{aligned}
$$

where $C$ is a positive constant independent of $N$. Hence, we let $N \rightarrow \infty$ in (3.6) to obtain a contradiction. This shows that when $s=-3 / 4$ and $b=1 / 2$, the estimate (3.1) fails.

In the same way as above, we can prove the rest of Theorem 1 (ii). In fact, the proof of Theorem 1.4 (iii) in [11] implies that if the estimate (1.3) holds with $s=-3 / 4$, we must have $b=1 / 2$ (see the relations (4.32) and (4.35) in [11]). Therefore, we have only to consider the case of $s=-3 / 4$ and $b=1 / 2$. We next note by the duality argument and the Plancherel theorem that the estimate (1.3) is equivalent to the following:

$$
\langle\hat{w}, \hat{\bar{u}} * \hat{\bar{v}}\rangle \leq C\|w\|_{Y_{1-b,-s}}\|u\|_{Y_{b, s}}\|v\|_{Y_{b, s}} .
$$

In this case, if we put $\hat{\bar{u}}_{1}=\hat{u}, \hat{\bar{v}}_{1}=\hat{v}$ and $\hat{w}_{1}=\hat{w}$ for functions $\hat{u}, \hat{v}$ and $\hat{w}$ defined as above, these three functions $\hat{\bar{u}}_{1}, \hat{\bar{v}}_{1}$ and $\hat{w}_{1}$ yield a contradiction to the estimate (3.7) with $s=-3 / 4$ and $b=1 / 2$.

Proof of Theorem 1 (iii). We show that if $a \geq-1 / 2$, then for any $\alpha, \beta \in \mathbf{R}$ the following estimate fails:

$$
\|u \bar{v}\|_{Y_{a,-1 / 4}} \leq C\|u\|_{Y_{\alpha,-1 / 4}}\|v\|_{Y_{\beta,-1 / 4}},
$$

which is stronger than Theorem 1 (iii) as is stated in Remark 1 (ii). We first note by the duality argument and the Plancherel theorem that the estimate (3.8) is equivalent to the following:

$$
\langle\hat{w}, \hat{u} * \hat{\bar{v}}\rangle \leq C\|w\|_{Y_{-a, 1 / 4}}\|u\|_{Y_{\alpha,-1 / 4}}\|v\|_{Y_{\beta,-1 / 4}} .
$$

Let $N$ be a sufficiently large positive number again. Let $\hat{u}$ and $\hat{v}$ be defined as in the above proof of Theorem 1 (i)-(ii). We choose $\hat{u}_{1}=\hat{u}$ and $\hat{\bar{v}}_{1}=\hat{v}$. We put

$$
\hat{w}_{1}(\tau, \xi)= \begin{cases}1, & |\tau+2 N \xi| \leq 1 / 2, \quad|\xi| \leq 1, \\ 0, & \text { otherwise. }\end{cases}
$$

Let $A=\{(\tau, \xi) ;|\tau+2 N \xi| \leq 1 / 10, \quad|\xi| \leq 1 / 10\}$. By the definitions of $\hat{u}_{1}$ and $\hat{\bar{v}}_{1}$, we easily see that for each $\left(\tau^{\prime}, \xi^{\prime}\right) \in A$, the support of $\hat{u}_{1}\left(\tau^{\prime}-\tau, \xi^{\prime}-\xi\right)$ intersects 
effectively the support of $\hat{\bar{v}}_{1}(\tau, \xi)$. Therefore, we have

$$
\left(\hat{u}_{1} * \hat{\bar{v}}_{1}\right)(\tau, \xi) \sim 1, \quad(\tau, \xi) \in A .
$$

Accordingly, since supp $\hat{w}_{1} \supset A$, we obtain

$$
\left\langle\hat{w}_{1}, \hat{u}_{1} * \hat{\bar{v}}_{1}\right\rangle \geq C|A| \sim 1 \text {. }
$$

On the other hand, simple calculations yield

$$
\begin{aligned}
& \left\|w_{1}\right\|_{Y_{-a, 1 / 4}} \sim\left[\left|\operatorname{supp} \hat{w}_{1}\right| \times N^{-2 a}\right]^{1 / 2} \sim N^{-a}, \\
& \left\|u_{1}\right\|_{Y_{\alpha,-1 / 4}}, \quad\left\|v_{1}\right\|_{Y_{\beta,-1 / 4}} \sim N^{-1 / 4} .
\end{aligned}
$$

Here we note that the power of $N$ in the right hand side of (3.12) does not depend on $\alpha$ and $\beta$. If the estimate (3.9) holds, then we must have by (3.10)-(3.12)

$$
1 \leq C N^{-a} \times\left(N^{-1 / 4}\right)^{2}=C N^{-a-1 / 2}
$$

where $C$ is a positive constant independent of $N$. Since $N$ is an arbitrary large positive integer, we conclude by (3.13) that the following relation must hold:

$$
a \leq-1 / 2 \text {. }
$$

Thus, it remains only to exclude the case of $a=-1 / 2$. We change the function $w_{1}$ to another one, while we leave $u_{1}$ and $v_{1}$ as they are. Let $\eta$ be a sufficiently small positive number independent of $N$. We next put

$$
\hat{w}_{2}(\tau, \xi)=\left\{\begin{array}{l}
(1+\tau)^{-1}, \quad|\tau+2 N \xi| \leq \eta, \quad 1 \leq \tau \leq \eta N \\
0, \quad \text { otherwise }
\end{array}\right.
$$

Then, we have by the definitions of $\hat{w}_{2}, \hat{u}_{1}$ and $\hat{\bar{v}}_{1}$

$$
\begin{gathered}
\left\langle\hat{w}_{2}, \hat{u}_{1} * \hat{\bar{v}}_{1}\right\rangle \sim \int_{1}^{\eta N}(1+\tau)^{-1} \int_{-\tau /(2 N)-\eta /(2 N)}^{-\tau /(2 N)+\eta /(2 N)} d \xi d \tau \\
\sim N^{-1} \int_{1}^{\eta N}(1+\tau)^{-1} d \tau \sim N^{-1} \log N .
\end{gathered}
$$

On the other hand, since $|\xi| \leq C$ for $(\tau, \xi) \in$ supp $\hat{w}_{2}$, a simple calculation yields

$$
\begin{aligned}
\left\|w_{2}\right\|_{Y_{1 / 2,1 / 4}} \sim & \left(\int_{1}^{\eta} \int_{-\tau /(2 N)-\eta /(2 N)}^{-\tau /(2 N)+\eta /(2 N)}(1+\tau)^{-2}(1+|\xi|)^{1 / 2}\right. \\
& \left.\times\left(1+\left|\tau-\xi^{2}\right|\right) d \xi d \tau\right)^{1 / 2} \\
& \sim N^{-1 / 2}\left(\int_{1}^{\eta N}(1+\tau)^{-1} d \tau\right)^{1 / 2} \\
& \sim N^{-1 / 2}(\log N)^{1 / 2}
\end{aligned}
$$

Therefore, if the estimate (3.9) holds with $a=-1 / 2$, we must have by (3.12), (3.14) and (3.15)

$$
\begin{aligned}
N^{-1} \log N & \leq C N^{-1 / 2}(\log N)^{1 / 2} \times\left(N^{-1 / 4}\right)^{2} \\
& =C N^{-1}(\log N)^{1 / 2}
\end{aligned}
$$


where $C$ is a positive constant independent of $N$. We let $N \rightarrow \infty$ in (3.16) to obtain a contradiction. This completes the proof of Theorem 1 (iii).

\section{REFERENCES}

[1]. D. Bekiranov, T. OgaWA and G. Ponce, Weak solvability and well posedness of the coupled Schrödinger Korteweg-de Vries equations in the capillary-gravity interaction waves, Proc. Amer. Math. Soc., 125 (1997), pp. 2907-2919.

[2] D. Bekiranov, T. Ogawa and G. Ponce, Interaction equations for short and long dispersive waves, J. Funct. Anal., 158 (1998), pp. 357-388.

[3] J. Bourgain, Fourier restriction phenomena for certain lattice subsets and applications to nonlinear dispersive equations. I Schrödinger equations, Geom. Funct. Anal., 3 (1993), pp. 107-156.

[4] J. BouRgaIN, Fourier restriction phenomena for certain lattice subsets and applications to nonlinear dispersive equations. II The KdV equation, Geom. Funct. Anal., 3 (1993), pp. 209-262.

[5] J. Bourgain and J. Colliander, On well-posedness of the Zakharov system, Int. Math. Res. Not. (1996), pp. 515-546.

[6] J. GiniBre, Le problème de Cauchy pour des EDP semi-linéaires périodiques en variables d'espace (d'après Bourgain), Séminaire Bourbaki no. 796, Astérisque, 237 (1996), pp. 163-187.

[7] J. Ginibre, Y. Tsutsumi and G. Velo, On the Cauchy problem for the Zakharov system, J. Funct. Anal., 151 (1997), pp. 384-436.

[8] M. KEEL AND T. TAO, Local and global well-posedness of wave maps on $\mathbf{R}^{1+1}$ for rough data, Int. Math. Res. Not. (1998), pp. 1117-1156.

[9] C. E. Kenig, G. Ponce And L. Vega, The Cauchy problem for the Korteweg-de Vries equation in Sobolev spaces of negative indices, Duke Math. J., 71 (1993), pp. 1-21.

[10] C. Kenig, G. Ponce and L. Vega, A bilinear estimate with applications to the KdV equation, J. Amer. Math. Soc., 9 (1996), pp. 573-603.

[11] C. Kenig, G. Ponce and L. Vega, Quadratic forms for the 1-D semilinear Schrödinger equation, Trans. Amer. Math. Soc., 348 (1996), pp. 3323-3353.

[12] S. Klainerman AND M. Machedon, Space time estimates for null forms and the local existence theorem, Comm. Pure Appl. Math., 46 (1993), pp. 1221-1268.

[13] S. Klainerman and M. Machedon, Smoothing estimates for null forms and applications, Duke Math. J., 81 (1995), pp. 96-103.

[14] S. Klainerman and M. Machedon, Estimates for null forms and the space $H_{s, \delta}$, Int. Math. Res. Not. (1996), pp. 853-365.

[15] S. Klainerman AND S. SelBerg, Remark on the optimal regularity for equations of wave map in 3D, Comm. Part. Diff. Eqs., 22 (1997), pp. 901-918.

[16] H. LindBlad, A sharp counterexample to the local existence of low regularity solutions to nonlinear wave equations, Duke Math. J., 72 (1993), pp. 503-539.

[17] H. Lindblad, Counterexamples to local existence for semi-linear wave equations, Amer. J. Math., 118 (1996), pp. 1-16.

[18] J. RAUCH AND M. REED, Nonlinear microlocal analysis of semilinear hyperbolic systems in one space dimension, Duke Math. J., 49 (1982), pp. 397-475.

[19] T. Ozawa, K. Tsutaya and Y. Tsutsumi, Well-posedness in energy space for the Cauchy problem of the Klein-Gordon-Zakharov equations with different propagation speeds in three space dimensions, Math. Annalen, 313 (1999), pp. 127-140.

[20] T. Ozawa, K. Tsutaya and Y. Tsutsumi, On the coupled system of nonlinear wave equations with different propagation speeds, in Proceedings of the conference "Evolution Equations: Existence, Regularity and Singularities", Banach Center Publications, Warsaw, 52 (2000), pp. 181-188.

[21] G. PONCE AND T. SIDERIS, Local regularity of nonlinear wave equations in three space dimensions, Comm. Part. Diff. Eqs., 18 (1993), pp. 169-177.

[22] G. Staffilani, Quadratic forms for a 2-D semilinear Schrödinger equation, Duke Math. J., 
86 (1997), pp. 79-107.

[23] R. S. StRICHARTZ, Restrictions of Fourier transforms to quadratic surfaces and decay of solutions of wave equations, Duke Math. J., 44 (1977), pp. 705-714.

[24] H. TAKAOKA, Well-posedness for the one dimensional nonlinear Schrödinger equation with the derivative nonlinearity, Adv. Diff. Eqns., 4 (1999), pp. 561-580.

[25] H. TAKaOKa And N. TzvetKov, On the local regularity of the Kadomtsev-Petviashvili-II equation, Int. Math. Res. Not. (2001), pp. 77-114.

[26] D. TAtaru, Local and global results for wave maps I, Comm. Part. Diff. Eqs., 23 (1998), pp. 1781-1793.

[27] P. A. Tomas, Restriction theorems for the Fourier transform, Proc. Symp. Pure Math., 35, Part I (1979), pp. 111-114.

[28] N. TzvetKov, On the Cauchy problem for Kadomtsev-Petviashvili equation, Comm. Part. Diff. Eqs., 24 (1999), pp. 1367-1397.

[29] K. Tsugawa, Well-posedness in the energy space for the Cauchy problem of the coupled system of complex scalar field and Maxwell equations, Fukcialaj Ekvacioj, 43 (2000), pp. 127-161.

[30] Y. ZHOU, Local existence with minimal regularity for nonlinear wave equations, Amer. J. Math., 119 (1997), pp. 671-703. 